\title{
DIELECTRIC MONITORING OF THE PAN FIBER STABILIZATION PROCESS
}

\author{
J. Hofele ${ }^{1}$, M. Jung ${ }^{2}$, G. Link ${ }^{1}$ and J. Jelonnek ${ }^{1,3}$
}

\author{
$I H M^{1}, I H E^{3}$, Karlsruhe Institute of Technology (KIT), 76131 Karlsruhe, Germany \\ Korea Institute of Carbon Convergence Technology ${ }^{2}$ (KCTECH), Jeonju-si, 54853 Korea \\ julia.hofele@kit.edu
}

Keywords: dielectric measurements, microwave heating, in-situ monitoring, PAN fiber, stabilization, carbon fiber production

\begin{abstract}
The production of carbon fibers based on Polyacrylonitrile (PAN) precursor fibers is a very energy intensive process. In order to increase the energy efficiency, microwave plasma processes and, alternatively, dielectric heating with microwaves are in the focus. In this latter case, the knowledge of the dielectric properties over temperature is the key in the design of an appropriate microwave heating system. A measurement system was developed that is capable to monitor in-situ the change of the dielectric properties during the stabilization process of the PAN fiber. First results show that the loss tangent of the PAN fiber is strongly varying with temperature. Additionally, the dielectric properties are changing during the stabilization process due to the chemical transformation.
\end{abstract}

\section{Introduction}

Carbon fiber composites are the key components of future lightweight applications. But, due to the energy intensive production of carbon fibers, the final material costs are not competitive if compared to steel or aluminum even though the mechanical properties are superior [1]. Sunter et al. specify the practical minimum energy needed for the carbon fiber production to be $330 \mathrm{MJ} / \mathrm{kg}$ [2] whereas Fruehan et al. state $10 \mathrm{~kJ} / \mathrm{kg}$ for steel [3], respectively. The production process of carbon fibers starts from spinning the Polyacrylonitrile (PAN) fiber, which is then passing two major stages: the initial stabilization and the final carbonization stage. During the stabilization stage, different chemical reactions take place such as oxidation, cyclization and dehydration. Depending on the chemical composition, the reactions take place at temperatures between $180^{\circ} \mathrm{C}$ and $280^{\circ} \mathrm{C}$. The chemical transformation is an exothermic chemical reaction that has to be considered in the control of the heating rates and holding times. The most significant energy saving is expected at the stabilization stage [4]. Hence, new energy efficient production processes for the stabilization stage are necessary. Microwave heating might be one of the solutions. In [5] Zhang et al. compare microwave and conventional heating of PAN fibers. They state that microwave heating has a shortening influence on the reaction time and improves the fiber surface. In [5] the fibers are attached to a ceramic rod but no dielectric properties are provided neither for the ceramic rod nor for the pan fibers. It can be assumed that the microwave was predominantly heating the ceramic rod, which heated the fiber via thermal conduction. In [6] dielectric properties of PAN fibers are presented, but only to a limited range in temperature and also without information about the final stabilization degree, as the cooling down was not recorded.

For the successful design of an appropriate direct heating system, the knowledge of the temperature-dependent dielectric properties of the raw material together with the chemical process during the production is mandatory. In the following, a measurement system to 
measure the temperature dependent dielectric properties during the complete stabilization stage and its results are described.

\section{Experimental Setup}

The complete measurement system is shown in Fig. 1. The measurement is performed in a cylindrical TM010-mode cavity using the perturbation method at $2.45 \mathrm{GHz}$ with a quality factor of the empty cavity larger than 15000 . A $12 \mathrm{k}$ PAN fiber bundle is located in a quartz tube that is placed along the maximum electric field of the TM010-mode. A HP 8720D Network Analyzer measures the resonance frequency and quality factor of the system.

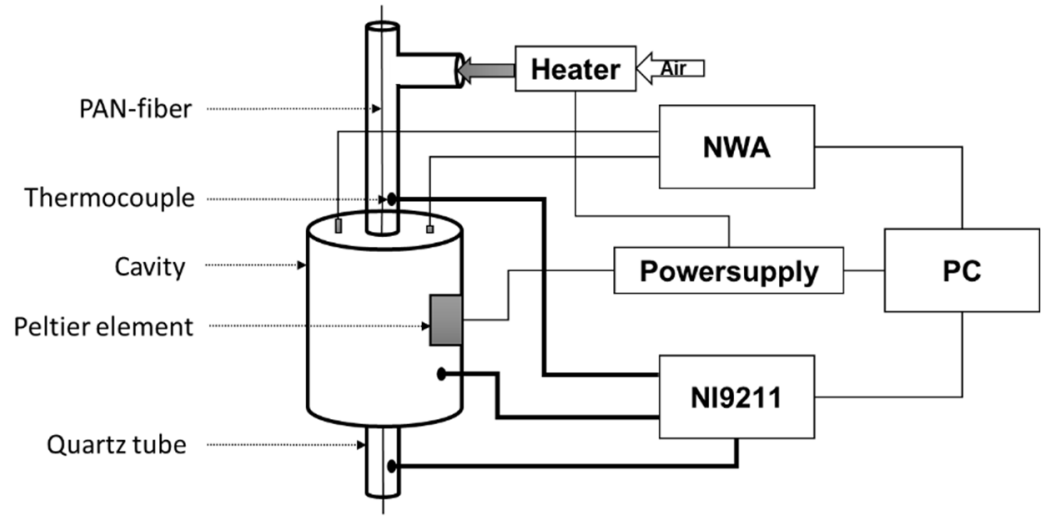

Figure 1. Measurement setup

The dielectric properties are calculated from the following equations [7]:

$$
\begin{aligned}
& \varepsilon^{\prime}=\frac{1}{A} \frac{f_{\text {ref }}-f_{s}}{f_{\text {ref }}} \frac{V_{c}}{V_{s}}+1 \\
& \tan \delta=\frac{1}{B} \frac{Q_{\text {ref }}-Q_{s}}{Q_{\text {ref }} Q_{s}} \frac{V_{c}}{V_{s}}
\end{aligned}
$$

where $\varepsilon^{\prime}$ is the relative dielectric constant, $\tan \delta$ is the relative dielectric loss tangent, $f_{\text {ref }}$ and $\mathrm{f}_{\mathrm{s}}$ are the unperturbed and perturbed resonance frequencies, respectively. $\mathrm{Q}_{\mathrm{ref}}$ and $\mathrm{Q}_{\mathrm{s}}$ are the unperturbed and perturbed quality factors. $V_{c}$ and $V_{s}$ are the volumes of the cavity and the sample. The calibration factors A and B depend on the sample and cavity geometries as well as on the final cavity wave pattern. The calibration of A and B is done with the help of CST Microwave Studio within the range of the expected dielectric properties.

For the temperature dependent measurements a heater, type MK-45R from Zinser GmbH, Germany is used to heat the air and was modified in order to use an analog control for the heater power. Pressurized air of one bar is used as input air flow. The air is heated up as it passes the heating cartridge and is channeled into the quartz tube where it heats the PAN fiber. A NI-9211 thermocouple input module is used to measure the temperature by use of type $\mathrm{K}$ thermocouples with an accuracy of $+/-2.2{ }^{\circ} \mathrm{C}$ and a measurement sensitivity of $0.07{ }^{\circ} \mathrm{C}$. Two thermocouples are placed in the quartz tube at the entry and the exit point of the cavity to measure the air flow temperature. A third one is used to measure the cavity 
temperature. Using a $180 \mathrm{~W}$ Peltier element the cavity temperature can be stabilized to a preset value of typically $20^{\circ} \mathrm{C}$ with an accuracy of $+/-0.3{ }^{\circ} \mathrm{C}$.

The accuracy of the measured dielectric properties is influenced by the following main parameters: temperature of the cavity and quartz tube, positioning of the quartz tube, simulation accuracy, NWA measurement, estimation of the sample volume.

Since the cavity perturbation with the PAN fiber is rather small, the precise measurement of the dielectric constant depends strongly on the accuracy of the measured resonance frequency of the empty cavity. This can be significantly improved by stabilizing the cavity temperature to $20^{\circ} \mathrm{C}$. A change in the quartz temperature leads to a frequency shift. Thus to distinguish the effect of the PAN fibers from the effect of the quartz tube, the temperature dependent frequency shift of an empty quartz tube is measured separately for each set of process parameters and used as reference. The impact of the quartz tube position is minimized by using the junction of the quartz tube as a stopping mechanism when inserted into the cavity. The simulation error can be neglected compared to the error from the sample volume and the NWA measurement.

Over all known errors the permittivity $\varepsilon$ is measured with an accuracy of $+/-0.65$ and the loss tangent $\tan \delta$ with $+/-0.001$ for the virgin PAN fiber at room temperature. During the stabilization process, the accuracy drops with increasing temperature. The worst case for the accuracy of the permittivity is $+/-2.1$ and $+/-0.06$ for the loss tangent. The stabilized fiber is measured at room temperature with an accuracy of $+/-1.3$ and $+/-0.02$, respectively. During the heating routine, the loss tangent increases which leads to the decline of the accuracy. This is due partly to the current measurement speed. The accuracy for the whole process improves when a fitting for the measurement curve is used, but this will extend the measurement time. It is preferred to measure faster in order to get a more precise temperature control. In a next step, it would be possible to switch to a more accurate analysis.

As a first test, the temperature is measured along the length of the cavity. In Fig. 2, the results of three consecutive runs show a temperature drop along the length due to heat losses to the Teflon insulation ring, the cavity wall, the quartz tube and the surroundings. The temperature drop inside the cavity is in the range of $10^{\circ} \mathrm{C}$. During the dielectric measurement, the thermocouples can not be placed too close to the inside of the cavity, as the metal tip is perturbing the electric field. In order to be sure to get the final temperature inside the cavity, a $10^{\circ} \mathrm{C}$ higher final temperature is selected. 


\section{High Frequency Heating}

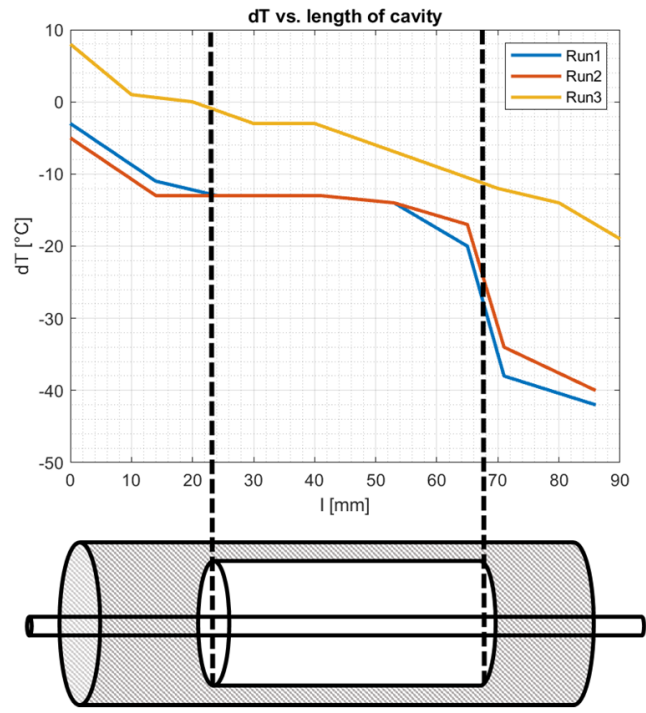

Figure 2. Temperature along the length of the cavity

\section{Experimental Results}

According to the DSC measurements, to be seen in Fig. 3, the stabilization process of the PAN fibers starts between $220-240{ }^{\circ} \mathrm{C}$. Two local maxima can be distinguished. As reported by Heine [8], the first peak can be assigned to the cyclisation, whereas the second peak is a combination of dehydration and oxidation. Thus the different reactions of which the stabilization process consists, start at different temperatures. The DSC measurements also show, that the heating rate as an influence on the heat flow produced by the exothermic reaction.

A process parameter set for the dielectric properties analysis consists of a heating rate, final temperature and holding time. The temperature measured by the thermocouple at the entry point of the cavity is defined as final temperature, as the heater control is using it as input. The sets, which are chosen based on the DSC measurements, are noted in Table 1. The temperature, resonance frequency and quality factor are measured every two seconds.

Table 1. Process parameter sets used for dielectric measurements

\begin{tabular}{cccccccc}
\hline $\begin{array}{c}\text { Process } \\
\text { Parameter Set }\end{array}$ & $\mathbf{1}$ & $\mathbf{2}$ & $\mathbf{3}$ & $\mathbf{4}$ & $\mathbf{5}$ & $\mathbf{6}$ & $\mathbf{7}$ \\
\hline $\begin{array}{c}\text { Heating Rate } \\
{\left[{ }^{\circ} \mathrm{C} / \mathrm{min}\right]}\end{array}$ & 10 & 5 & 2 & 10 & 10 & 10 & 10 \\
\hline $\begin{array}{c}\text { Final Temperature } \\
{\left[{ }^{\circ} \mathrm{C}\right]}\end{array}$ & 260 & 260 & 260 & 280 & 300 & 260 & 260 \\
\hline $\begin{array}{c}\text { Holding Time } \\
{[\mathrm{min}]}\end{array}$ & 50 & 50 & 50 & 50 & 50 & 75 & 170 \\
\hline
\end{tabular}




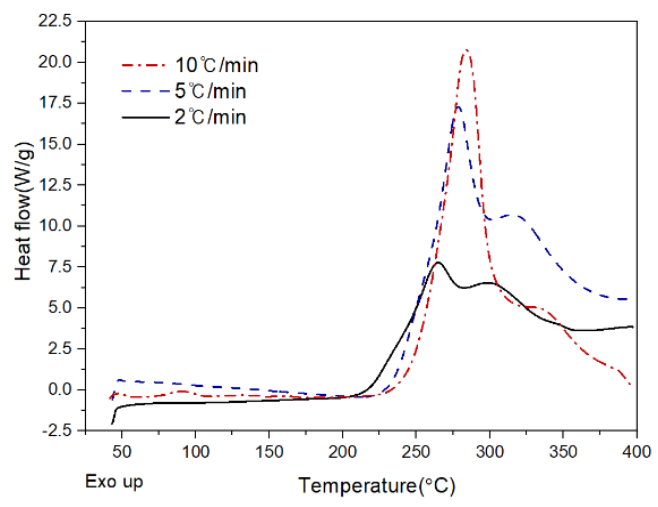

Figure 3. DSC measurement results of PAN fiber for different heating rates $\mathrm{CKCTECH}$

a) Varying holding time, Time dependent
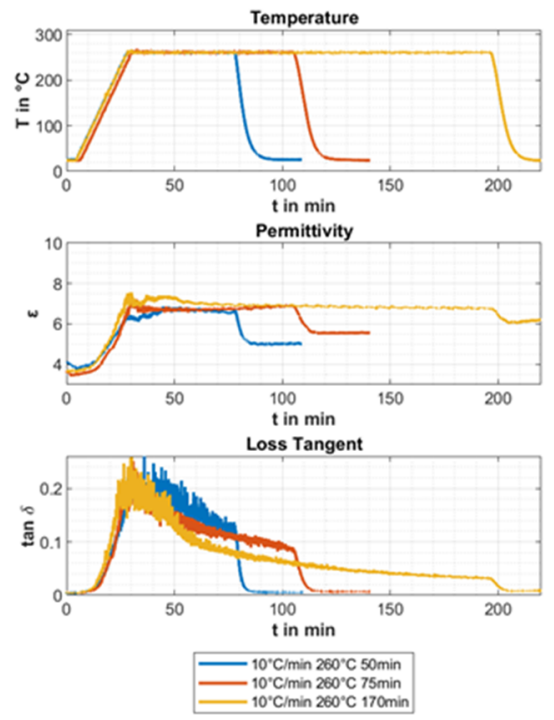

b) Varying holding time, Temperature dependent
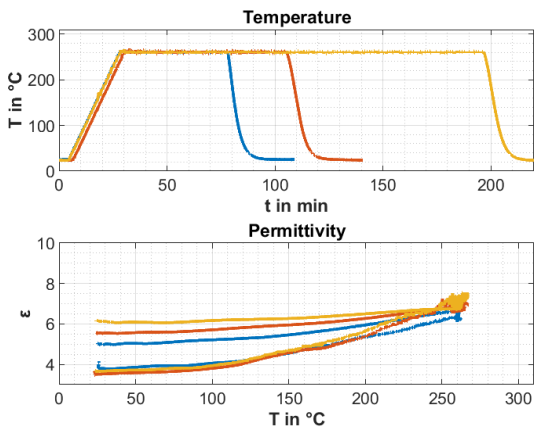

Loss Tangent

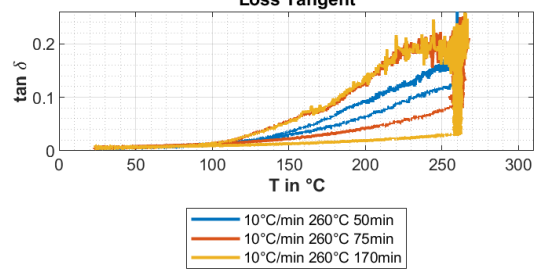

Figure 4. Dielectric properties for different measurement sets versus time and temperature 
c) Varying heat rate, Temperature dependent

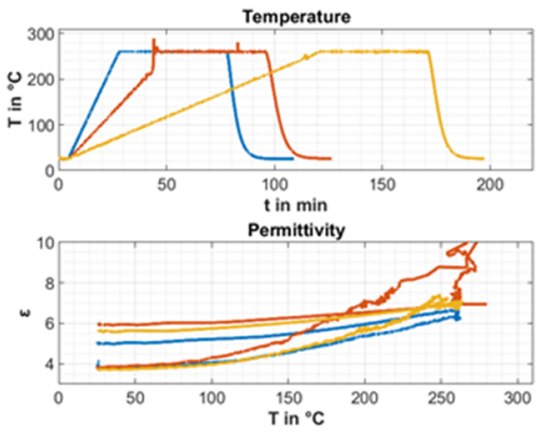

Loss Tangent

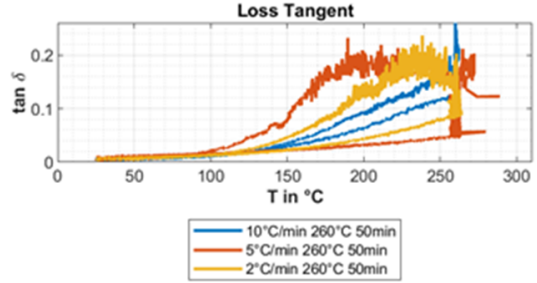

d) Varying final temperature, Temperature dependent
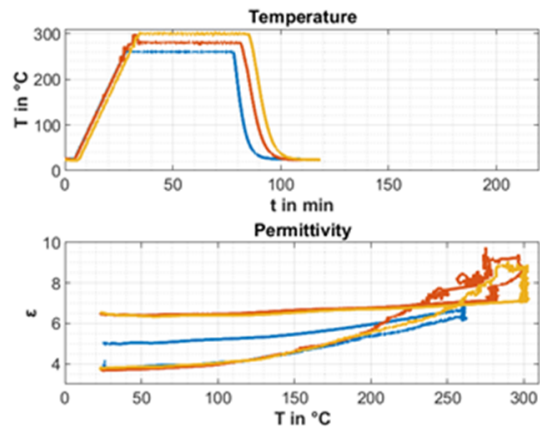

Loss Tangent

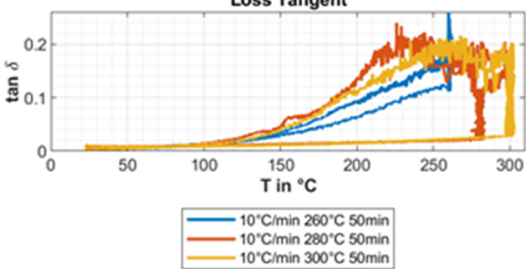

Figure 4. Dielectric properties for different measurement sets versus time and temperature

A strong temperature dependence can be seen for both the loss tangent and the permittivity in Fig. 4 respectively. As heating rate, final temperature and holding time have a great influence on the chemical reaction, they also affect the dielectric properties. Considering all process parameter sets, it can be said that the permittivity and loss tangent increase with an increasing temperature. The permittivity rises only about to the double value, whereas the loss tangent rises to about the twentyfold value. Both decrease again with the ongoing chemical reaction that unfolds during the holding time. The dielectric properties after the stabilization vary for different heating rates, final temperatures and holding times due to different stabilization degrees.

A heating rate of about $10^{\circ} \mathrm{C} / \mathrm{min}$ leads to a smaller change in loss tangent and permittivity compared to slower heating rates. This is may be due to the shorter overall process time. For increasing heating rates, the holding time has to be longer or the final temperature higher in order to achieve the same result. The correspondent values can be found in Table 2 . The process parameter set " 0 " contains the information of the untreated virgin PAN fiber. The varying stabilization degrees are also evident from the densities. It is shown in Table 2. Literature values for stabilized PAN fibers of different chemical compositions are in the range from $1.34 \mathrm{~g} / \mathrm{cm}^{3}$ to $1.39 \mathrm{~g} / \mathrm{cm}^{3}$ [9] (SGL Carbon). Similar densities result for the process parameter sets " 3 " and " 6 ". For the dielectric properties the only publication were found in [6], however the chemical composition is unknown, hence, a direct comparison is not possible.

A final temperature higher than $260^{\circ} \mathrm{C}$ leads to a higher dielectric permittivity, but no changes in the permittivity can be found when increased more, whereas the loss tangent is highest for the highest end temperature. 
Table 2. Comparison of the properties of virgin PAN and stabilized fibers

\begin{tabular}{ccccccccc}
\hline $\begin{array}{c}\text { Process Parameter } \\
\text { Set }\end{array}$ & $\mathbf{0}$ & $\mathbf{1}$ & $\mathbf{2}$ & $\mathbf{3}$ & $\mathbf{4}$ & $\mathbf{5}$ & $\mathbf{6}$ & $\mathbf{7}$ \\
\hline Heating Rate $\left[{ }^{\circ} \mathrm{C} / \mathrm{min}\right]$ & - & 10 & 5 & 2 & 10 & 10 & 10 & 10 \\
\hline Final Temperature $\left[{ }^{\circ} \mathrm{C}\right]$ & - & 260 & 260 & 260 & 280 & 300 & 260 & 260 \\
\hline Holding Time $[\mathrm{min}]$ & - & 50 & 50 & 50 & 50 & 50 & 75 & 170 \\
\hline Density $\left[\mathrm{g} / \mathrm{cm}^{3}\right]$ & 1,17 & 1,27 & 1,41 & 1,36 & 1,42 & 1,46 & 1,37 & 1,45 \\
\hline Permittivity & 3,8 & 5,0 & 6,2 & 5,6 & 6,5 & 6,5 & 5,6 & 6,1 \\
\hline Loss tangent $\mathrm{x} 10^{-3}$ & 4 & 6 & 10 & 8 & 9 & 11 & 6,5 & 8,5 \\
\hline
\end{tabular}

\section{Conclusion}

A measurement system is presented, that allows to track the change of the dielectric properties of PAN fiber during the stabilization stage. The measurements during that process show a significant temperature dependency of the dielectric properties. More importantly, the measurement system allows to follow the chemical process during the stabilization stage of the PAN fiber. That gets visible from the decrease of the loss tangent and the permittivity during the process even though the final temperature is reached and kept constant. That follow-up in the change of the loss tangent will allow to recognize e.g. hot spots in the process and to determine the end of the stabilization stage. As different process parameter sets lead to varying dielectric properties, the next step will be to determine the degree of the stabilization precisely with the help of other measurement techniques such as FTIR. In addition, it is planned to compare the dielectric properties of industrial stabilized fibers to the current results. Clearly, the knowledge of the temperature dependent dielectric properties provides a much better view on to the transients in the chemical process during microwave heating of PAN fiber during the stabilization phase.

\section{Acknowledgment}

The authors acknowledge the financial support by the Federal Ministry for Economic Affairs and Energy of Germany in the project REINFORCE (project number ZF4204603SY7) and the DSC measurements done by the project partner $\mathrm{KCTECH}$.

\section{References}

1. Frank, E. et al., Angewandte Chemie International Edition, Wiley Online Library, 2014, vol. 53, 5262-5298.

2. Sunter, D. et al., The manufacturing energy intensity of carbon fiber reinforced polymer composites and its effect on life cycle energy use for vehicle door lightweighting, 20th International Conference on Composite Materials, 2015.

3. Fruehan R.J. et al., Theoretical Minimum Energies To Produce Steel for Selected Conditions, U.S. Department of Energy, 2000.

4. Liddell, H. et al., Bandwidth Study on Energy Use and Potential Energy Saving Opportunities in U.S. Carbon Fiber Reinforced Polymer Manufacturing. U.S. Department of Energy, 2017.

5. Zhang, C. et al., Comparison of microwave and conventional heating methods for oxidative stabilization of polyacrylonitrile fibers at different holding time and heating rate, Ceramics International, 2018, Vol. 44, Issue 12, 14377-14385. 
6. Paulauskas, F. L. et al., Temperature-Dependent Dielectric Measurements of Polyacrylonitrile Fibers During Air, SAMPE 2004 Materials and Processing Technology.

7. Chen, L. F., Microwave Electronics: Measurements and Material Characterization, 2004, J. Wiley \& Sons

8. Heine, M., Optimierung der Reaktionsbedingungen von thermoplastischen Polymerfasern zur Kohlenstoffaser-Herstellung am Beispiel von Polyacrylnitril, Dissertaion, 1989, Falk-Verlag Ötigheim

9. Takaku, A. et al., Tensile properties of carbon fibers from acrylic fibers stabilized under isothermal conditions. J. Appl. Polym. Sci.,1985, 30: 1565-1571. 